\title{
Laser Interferometric Displacement Measurements of Multi-Layer Actuators and PZT Ceramics
}

\author{
L. BURIANOVA,,${ }^{1, *}$ C. R. BOWEN, ${ }^{2}$ M. PROKOPOVA, ${ }^{1}$ \\ AND M. SULC ${ }^{1}$ \\ ${ }^{1}$ Technical University of Liberec, Department of Physics and International \\ Center for Piezoelectric Research, CZ-46117 Liberec, Czech Republic \\ ${ }^{2}$ University of Bath, Department of Mechanical Engineering, Bath, \\ BA2 7AY, UK
}

\begin{abstract}
This paper deals with the characterization of multi-layer actuators and bulk PZT ceramics under the influence of an applied electric field. The displacement profile of materials and devices with evaporated reflective dielectric layers was measured using a doublebeam laser interferometer at room temperature. The displacements of $3 \mathrm{~mm}$ thick plates of bulk soft PZT were characterized to determine bulk material properties. The tested multi-layer actuator devices consisted of 20 layers, with each layer being 87 micrometers thick. The results of "point to point scanning" of displacement across piezoelectric materials are presented and the effective actuation area of the actuator displacement is determined. Comparisons are made of the behaviour of the bulk ceramics and the actuator. In addition to experimental measurement, representative coupled-field finite element model was constructed to compare with experimental $x-y$ displacement data of multi-layer actuator.
\end{abstract}

Keywords PZT ceramics; Multi-layer actuator; Piezoelectric coefficient; Laser interferometry

PACS: $77.84 . \mathrm{D} ; 77.65 ; 42.87$

\section{Introduction}

It is well known that piezoelectric actuators designed as multi-layer or bimorph devices have many applications in both industry and academia. Multi-layer piezoelectric actuators, such as those based on lead zirconate titanate (PZT) thick films, have many advantages including high displacement for a low drive voltage, quick response time, large generative force and high electromechanical coupling. Multi-layer structures have been intensively investigated in order to improve their reliability and to expand their applications [1]. The technology of thin films and methods of piezoelectric measurements and characterisation have been well researched and developed [2,3]. However, due to the composite structure of the multilayer piezoelectric actuators and the electrode configuration, certain disadvantages such as fatigue and degradation of performance as a function of time have been observed [2, 4]. It has been shown that fatigue is due to the development of high internal stresses around the

Received September 6, 2004; In revised form January 20, 2005.

*Corresponding author. E-mail: lidmila.burianova@ vslib.cz 
active and inactive areas of the piezoelectric material at the electrode edges. The effect of delamination of the electrode layers during processing [4] can also lead to degradation of mechanical performance, such as the displacement-field characteristics. These issues lead to a need to develop non-destructive testing methods for piezoelectric devices, in particular direct measurement of the induced displacement. Different displacement measurement methodologies, based on the measurement of changes of capacitance, inductance (using LVDT sensors), velocity by Doppler principle and strain by gauges or fiber optic probes are known. However, laser interferometry is a common and widely used method for measurement of induced displacements [5-7]. In particular, the "point to point" scanning method or "out-of-plane displacements" contributions measured by Moiré interferometer [8] have an importance for this application.

In this paper, we present results obtained by a double beam Mach-Zehnder type interferometer, which has been used for characterization of the displacements induced by an applied electric field [2, 3, 7, 9, 10] for multi-layer actuators and PZT bulk ceramics. All measurements in this paper are provided at room temperature.

\section{Experimental Method}

\section{Mechanical Displacements Measured by Laser Interferometer}

A double-beam laser interferometer system has been used to measure the electric field induced surface displacements of:

(i) bulk ceramics samples (AC driving electric field), and

(ii) multi-layer actuators (DC driving electric field).

A stabilised He-Ne laser (SIOS Messtechnik, SL $-03, \lambda=632.8 \mathrm{~nm}$ ) was used for measurement of the small displacements of the bulk ceramic samples. It can be easily shown that, the displacement amplitude $d_{0}$ can be calculated [9]

$$
d_{0}=\frac{\lambda}{\pi \sqrt{2}} \frac{V_{\mathrm{out}}}{V_{\mathrm{p}-\mathrm{p}}},
$$

where voltage $V_{\text {out }}$ is the root mean squared (rms) value of the detected optical signal, and $V_{\mathrm{p}-\mathrm{p}}$ is the peak to peak value of voltage corresponding to the interference optical signal, which varies between the maximum and minimum light intensities.

The voltage response was detected using a lock-in amplifier (Stanford Research Systems, SR830 DSP) and by measuring the displacement $d_{0}$, the piezoelectric coefficients $d_{\mathrm{i} \lambda}$ can be calculated using Eq. (2).

$$
d_{i \lambda}=\frac{\partial S_{\lambda}}{\partial E_{i}}=\frac{d_{0}}{U_{m}} \cdot \frac{t}{x} .
$$

In Eq. (2), the symbol $S_{\lambda}$ denotes the strain tensor component, $E_{\mathrm{i}}$ components of electric field intensity, and $U_{\mathrm{m}}$ is the AC driving voltage amplitude. The other symbols denote the corresponding length $x$ in the direction of sample deformation and thickness $t$ of the sample in the direction of the applied electric field. The basic scheme of the double-beam interferometer, used for the bulk ceramics specimen measurements, was reported $[2,7,9]$. The larger displacements $\left(d_{0}>\lambda\right)$ of the multi-layer actuator were measured by a different method, as shown in Fig. 1. Under the influence of a DC triangle voltage, the multi-layer 


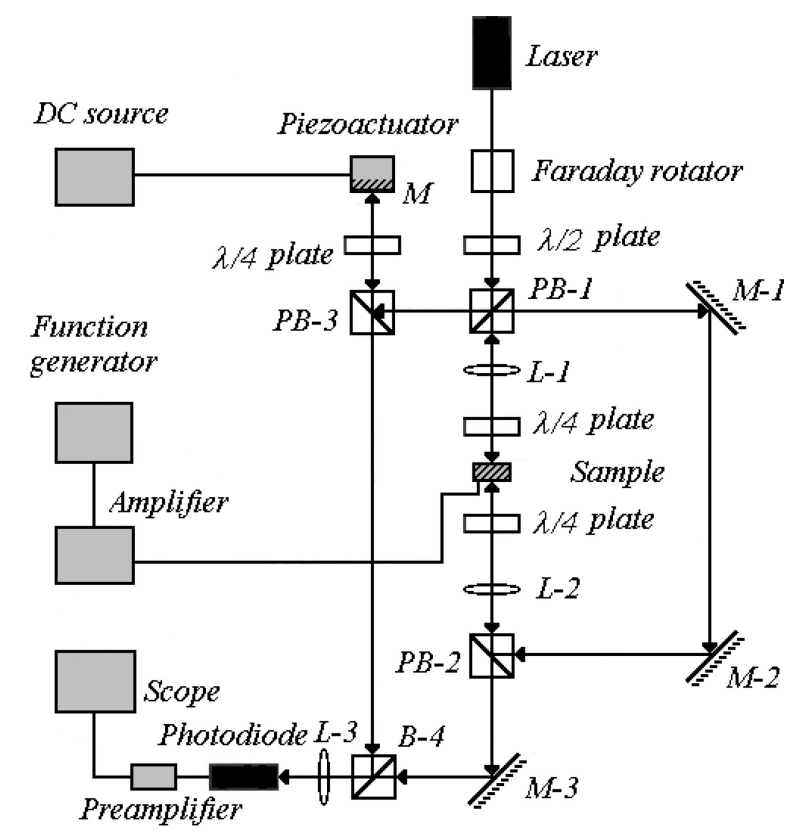

Legend:

$M$ - mirror

$L-$ lens

$B$ - beam splitter

$P B$ - polarized beam splitter

Figure 1. Scheme of the double-beam laser interferometer (measurements of the multi-layer actuator displacements).

actuator changes the length of the sample arm of the interferometer in accordance with the driving voltage. The frequency of the DC triangle voltage was $0.2 \mathrm{~Hz}$ and the voltage was without an offset. The time development of the intensity of the interference beam was detected by a photodiode and plotted by a Hewlett Packard 54600B oscilloscope. A Physik Instrumente actuator changed the reference arm length and corrected the position of the minimum and maximum of the photodiode signal (the displacement inflection). The time difference $\Delta \tau$, defined as the period when the intensity of the interfering light changed from a maximum to minimum, was measured. The phase is changed by $\pi$ in this time interval and corresponds to a change of the light path by $\lambda / 2$. Due to the fact the beam is reflected, the actuator displacement is $\lambda / 4$ in the time $\Delta \tau$. This displacement at the time $\Delta \tau$ is proportional to the voltage change $\Delta U$ on the sample, Eq. (3).

$$
\Delta U: \frac{\lambda}{4}=U_{d}: d_{0}
$$

$U_{\mathrm{d}}$ is the DC driving voltage amplitude and $d_{0}$ is the displacement of actuator surface. Eq. (3) implies that,

$$
\Delta U=\Delta \tau \frac{2 U_{d}}{T}=2 \cdot \Delta \tau \cdot f \cdot U_{d},
$$

where $T / 2$ is the time of increasing (decreasing) of driving voltage on the sample and $f$ is the frequency of triangle voltage on the sample. The displacement amplitude $d_{0}$ can be 
calculated from Eq. (5).

$$
d_{0}=\frac{\lambda}{8 f \cdot \Delta \tau},
$$

Since there are $N$ layers in the multi-layer actuator, the displacement $d_{0}$ of an individual layer is $d_{0} / N$. The $d_{33}$ piezoelectric coefficient of an individual layer was calculated using Eq. (6).

$$
d_{33}=\frac{d_{0}}{N \cdot U_{d}}=\frac{\lambda}{N \cdot 8 f \cdot \Delta \tau U_{d}} .
$$

\section{Samples Under Investigation and Testing Conditions}

We studied samples of APC850 'soft' bulk PZT ceramics provided by American Piezoceramics International, Mackeyville, PA, USA. Rectangular plate-shaped samples were $4 \times 3.5 \times 3 \mathrm{~mm}^{3}$. The samples were electroded on both major surfaces and poled in the thickness direction by the manufacturers. The thickness of electrode $t_{\mathrm{el}}=2 \mu \mathrm{m}$. The applied electric field, $E=1.7 \mathrm{kV} / \mathrm{m}$ and frequency, $f=1 \mathrm{kHz}$.

Multi-layer actuators, based on the thick layers of PZT-5H, were supplied by Morgan Matroc Unilator Division. The electrode geometry of a typical multi-layer actuator with an ineffective volume at the device edges is shown in Fig. 2a. The square shaped plate was $10 \times 10 \times 1.8 \mathrm{~mm}^{3}$. From an optical microscope section, Fig. 2b, the thickness of a single PZT layer (with internal electrode) $t_{1}=87 \mu \mathrm{m}$, number of layers $N=20$, with a total thickness of all layers $t_{\text {tot }}=1.8 \mathrm{~mm}$. The driving electric voltage $U_{\mathrm{d}}=90 \mathrm{~V}$, i.e $E=1.03 \mathrm{MV} / \mathrm{m}$ on each layer, with a frequency $f=0.2 \mathrm{~Hz}$.

To improve the reflectivity of the actuator surfaces for interferometry, reflective dielectric layers were evaporated on opposite surfaces of both multi-layer actuators and bulk PZT ceramics samples after grinding and polishing. The thickness of the dielectric layer $t_{\mathrm{R}}$ was $357 \mathrm{~nm}$ and consisted of several $\mathrm{TiO}_{2}$ and $\mathrm{SiO}_{2}$ thin layers. The reflective layers had a smaller thickness in the gaps and small troughs on the actuator surface due to its surface finish, but they remained reflective. The reflectance spectrum of the reflective dielectric layer evaporated on the bulk APC850 type PZT sample is shown in Fig. 3. The reflective layers were on surfaces perpendicular to the electrode layers, resulting in the reflective layers being applied to the bulk PZT surface, which deformed via the $d_{31}$ coefficient and to the surface, which deformed via the $d_{33}$ coefficient for the multi-layer actuator. The reflectance of the dielectric layer evaporated on the PZT ceramics APC 850 was over $27 \%$ for a laser beam with $\lambda=632.8 \mathrm{~nm}$. The reflectance of the dielectric layer on the multi-layer actuators was higher than on APC850, due to the better surface properties of the actuators and was typically $40 \%$. During displacement measurements the samples were clamped in a holder between a spherical contact and cylindrical ring (inside diameter $d_{\text {in }}=1.57 \mathrm{~mm}$, outside diameter $d_{\text {out }}=2.00 \mathrm{~mm}$ ).

\section{Multi-Layer Modelling}

Finite element modelling of the multi layer actuator displacement profile was conducted using coupled-field analysis via ANSYS 5.7. Since a high mesh density is required to model the changing electric field direction at the electrode edges, two layers of the multi layer actuator were modelled. The electrode spacing was $87 \mu \mathrm{m}$ with a $0.5 \mathrm{~mm}$ gap at the edge of 


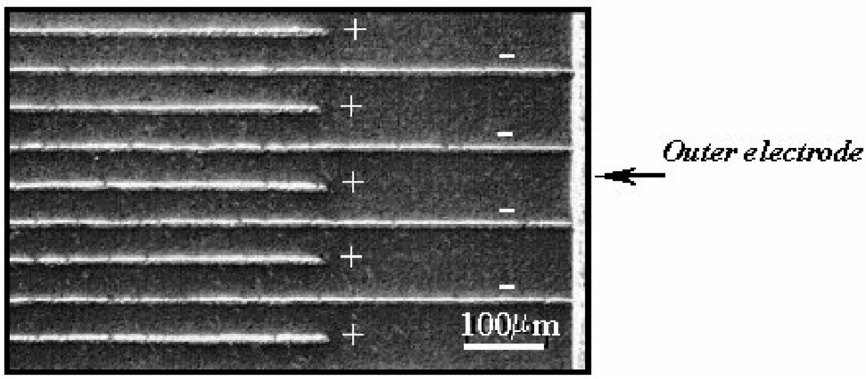

a)

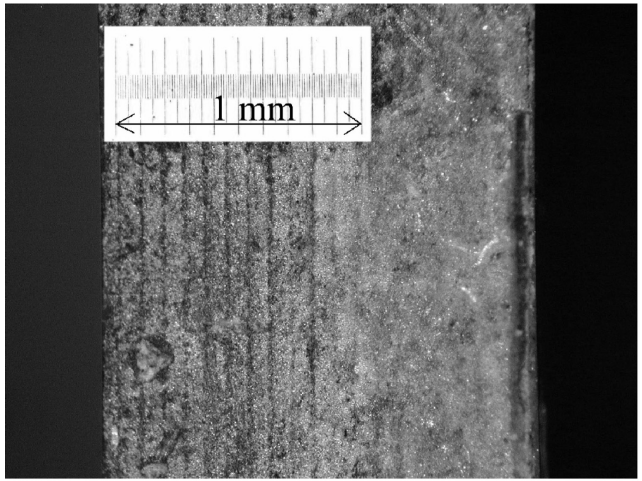

b)

Figure 2. a) Electrode geometry of a typical multi-layer actuator with an ineffective volume at the device edges (typically $0.5 \mathrm{~mm}$ ). b) Optical microscope image of actuator layers.

the model, as observed by optical microscopy of the multi layer actuator tested in this work (Fig. 4). Material properties (stiffness, piezoelectric and permittivity matrix) of PZT-5H were used [11], Fig. 5, and a three dimensional coupled-field element (SOLID 5) was used to model linear piezoelectric behaviour. Electrode 1 of the model in Fig. 4 was ground at a

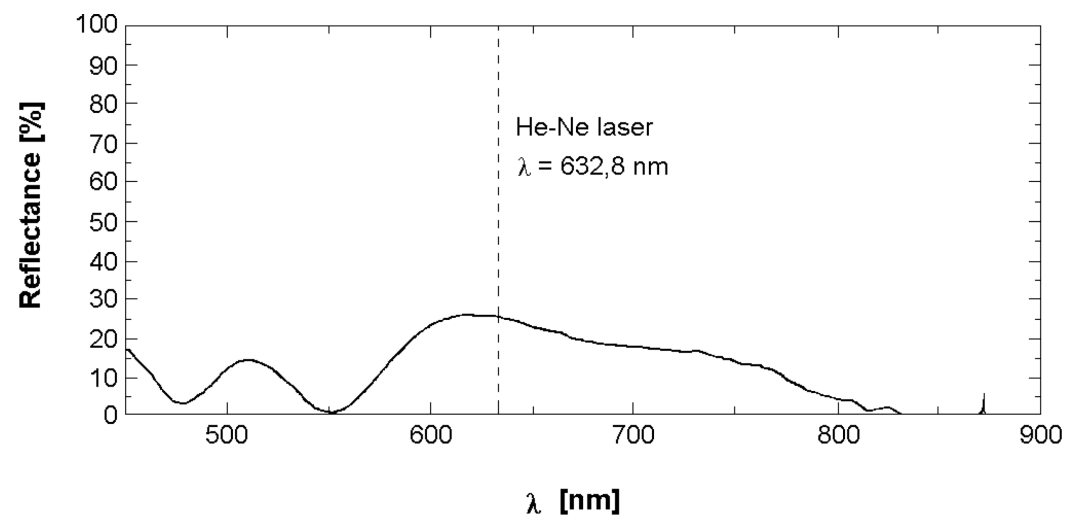

Figure 3. The reflectance spectrum of the thin dielectric layer on the sample face (APC850). 


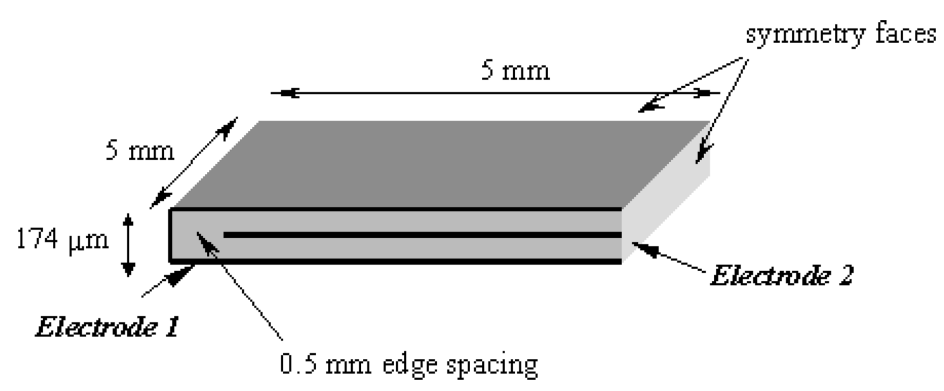

Figure 4. Finite element model of multi layer actuator. Dimensions and electrodes are indicated. The poling direction was reversed between the individual layers, as in the real device.

potential of $0 \mathrm{~V}$, while Electrode 2 was set to $90 \mathrm{~V}$ as in the experimental procedure. Since the polarisation direction alternates between individual layers in the multi layer, this was also undertaken in model. Once solved, the displacement profile was extracted from the model.

\section{Results and Discussion}

The basic mechanical response and displacement due to the applied electric field was measured for both the bulk PZT ceramics and for the multi-layer actuator samples. In addition to the displacement amplitude, the aim was to determine the effective surface area where the mechanical displacement is useful for actuation function.

The results of "point to point" scanning of displacement $d_{0}$ across area of displacement of piezoelectric ceramics (in an $y$-z manner) and of multi-layer actuator surface

$$
\begin{aligned}
& {[c]=\left[\begin{array}{cccccc}
12.7 & 8.0 & 8.5 & 0 & 0 & 0 \\
& 12.7 & 8.5 & 0 & 0 & 0 \\
& & 11.7 & 0 & 0 & 0 \\
& & & 2.3 & 0 & 0 \\
& & & & 2.3 & 0 \\
& & & & & 2.4
\end{array}\right] \times 10^{10} \mathrm{Nm}^{-2}} \\
& {[d]=\left[\begin{array}{ccc}
0 & 0 & -274 \\
0 & 0 & -274 \\
0 & 0 & 593 \\
0 & 0 & 0 \\
0 & 741 & 0 \\
741 & 0 & 0
\end{array}\right] x 10^{-12} \mathrm{CN}^{-1} \quad\left[\quad\left[\varepsilon_{0}^{5}\right]^{5}=\left[\begin{array}{ccc}
1700 & 0 & 0 \\
0 & 1700 & 0 \\
0 & 0 & 1470
\end{array}\right]\right.}
\end{aligned}
$$

Figure 5. Piezoelectric Material Parameters of PZT-5H [11] $[c]$ denotes the stiffness matrix, $[d]$ is piezoelectric strain matrix and $[\varepsilon]^{\mathrm{s}}=$ permittivity at constant strain matrix, where $\varepsilon_{o}=8.85 \cdot 10^{-12}$ $\mathrm{Fm}^{-1}$. The density of the piezoelectric material was $7500 \mathrm{~kg} \mathrm{~m}^{-3}$. The poling direction is in the $z$ direction for this set of materials data. 


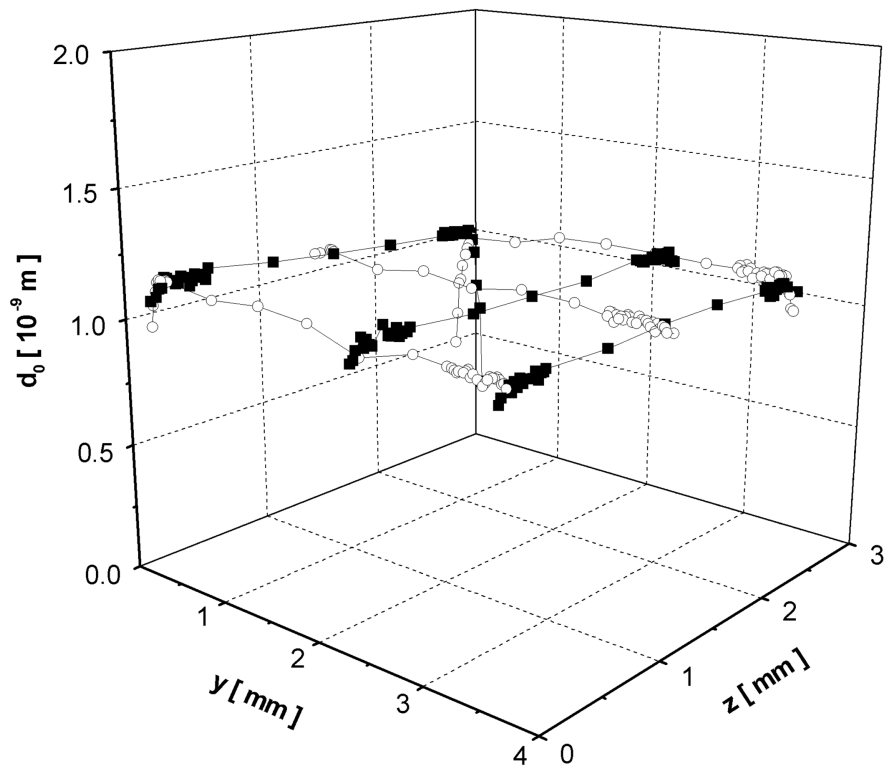

Figure 6. 3D graph of the measured displacement of bulk ceramics surface. Scanning in the horizontal (ם) and vertical (o) direction.

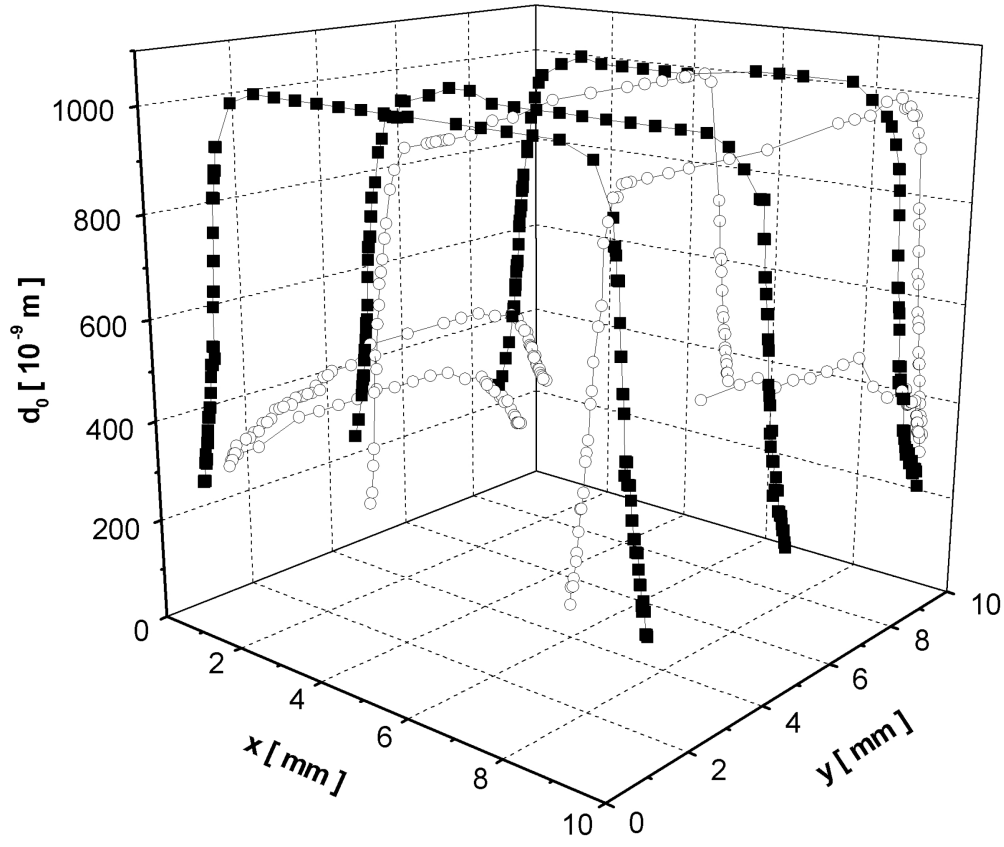

Figure 7. 3D graph of the measured displacement of multi-layer actuator surface. Scanning in the $\mathrm{x}(\boldsymbol{\square})-\mathrm{y}(\mathrm{O})$ plane. 
(in an $x-\mathrm{y}$ manner) are presented in the Figs. 6 and 7, respectively. No difference was observed between values of displacement determined in both scanning directions. The error of the displacement $d_{0}$ measurements was $2 \%$ and the high accuracy is associated with the use of a double beam laser interferometer and our experimental set-up, which eliminates the supplementary deformations of the samples. The drop in displacement at the multilayer actuator edge (see Fig. 7) is due to the inactive area associated with the electrode geometry. The effective actuation area was calculated for both the bulk PZT ceramics and for the multi-layer actuator using the measured values of displacement in Figs. 6 and 7. The "effective actuation area" is defined as the area where the displacement change is less then 5\%. A substantial drop in the surface displacement was observed $1.0 \mathrm{~mm}$ and $0.1 \mathrm{~mm}$ from edge of the multi-layer actuator and bulk ceramics, respectively. The amplitude of the displacement of the bulk PZT ceramics sample is approximately constant at $90 \%$ of the surface area (in the bounds of the experimental error). The effective active surface of the multi-layer actuator is reduced to $64 \%$ of the total sample surface (due to the inactive electrode edges). The values of displacement $d_{0}$ of effective active surface of actuators and bulk PZT ceramics were $1 \mu \mathrm{m}(90 \mathrm{~V}$ on the thickness $1.8 \mathrm{~mm})$ and $1.1 \mathrm{~nm}(5 \mathrm{~V}$ on the thickness $3 \mathrm{~mm}$ ), respectively. The value of effective piezoelectric coefficient of PZT- 5H (multi-layer actuator) $d_{33}=560.10^{-12} \mathrm{CN}^{-1}$ was calculated using the Eq. (6). The piezoelectric coefficient $d_{31}$ of PZT-APC850 (bulk ceramics) was measured. From the Eq. (2) was obtained $d_{31}=-190.10^{-12} \mathrm{CN}^{-1}$. The displacement profile of the finite element model of the multi layer actuator is shown in Fig. 8. As with the experimental measurements, there is an inactive region $1 \mathrm{~mm}$ from the device edge associated with the inactive electrode edges. The maximum displacement at the centre of the device is $1.05 \mu \mathrm{m}$ and is in excellent agreement with experimental measurements $(1 \mu \mathrm{m})$. Since the model does not include non-liner behaviour, which increases the $d_{33}$ of PZT-5H at the high fields used in the work, it would be expected that the model would underestimate the measured deflection. The close agreement observed here may be due to the fact that the model does not include other factors which lead to an additional decrease in the displacement of the real device, such as the constraint of the electrode material, incomplete poling, misaligned electrodes etc.

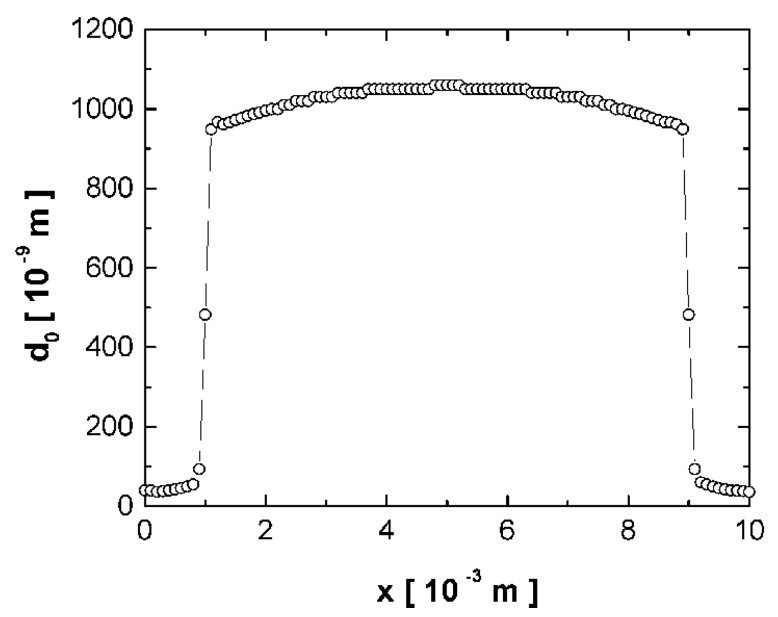

Figure 8. Calculated displacement profile of multi-layer actuator. Maximum displacement is $1.05 \mu \mathrm{m}$. 


\section{Conclusion}

The mechanical displacements of the active surface and the effective piezoelectric coefficients $d_{\mathrm{i} \lambda}$ of two types of actuators were measured via a double beam laser interferometer and an experimental set-up, which eliminates the supplementary deformations of the samples. The first of samples was a soft PZT bulk ceramic, the second was multi-layer actuator with PZT layers. The mechanical displacements of the active surface were measured "point to point" and three dimensional displacement profiles are presented. The effective active area of the PZT ceramics was $90 \%$ and the active area of multi layer actuator was $64 \%$. The effect of the inactive electrode edge in decreasing the effective active area of the actuator is shown and compared with finite element modelling, with good agreement. This method of actuator characterisation to determine piezoelectric coefficients $d_{\mathrm{i} \lambda}$, displacement $x-y$ profile and effective actuation area is important for the characterisation and development of actuators for industrial applications.

\section{Acknowledgments}

This work was supported by the Grant Agency of the Czech Republic (GACR 202/03/0569) and by the Ministry of Education of Czech Republic (Project Code MSM 242200002).

\section{References}

1. K. Uchino and S. Takahashi, Current Opinion in Solid State Materials Science 5, 698 (1996).

2. A. L. Kholkin, Ch. Wütchrich, D. V. Taylor, and N. Setter, Rev. Sci. Instrum. 67, 1935 (1996).

3. J. Nosek, L. Burianova, M. Sulc, C. Soyer, E. Cattan, and D. Remiens, Ferroelectrics 292, 103 (2003).

4. C. R. Bowen, S. Mahon, and M. L. Prieto, Ferroelectrics 241, 1843 (2000).

5. Q. M. Zhang, W. Y. Pan, and L. E. Cross, J. Appl. Phys. 63(8), 2492 (1988).

6. J. F. Li, P. Moses, and D. Viehland, Rev. Sci. Instrum. 66(1), 215 (1995).

7. W. Y. Pan and L. E. Cross, Rev. Sci. Instrum. 60(8), 2701 (1989).

8. H. Moilanen and S. Leppävuori, Sensors and Actuators A 92, 326 (2001).

9. L. Burianova, M. Sulc, and M. Prokopova, J. European Cer. Soc. 21, 1387 (2001).

10. W. Y. Pan, H. Wang, and L. E. Cross, Jap. J. Appl. Phys. 29, 1570 (1990).

11. H. A. Kunkel, S. Locke, and B. Pikeroen, IEEE Trans. Ultra. Ferro. Freq. Control. 37, 316 (1990). 